\title{
The importance of photographic gelatin
}

\author{
from Yves Lestra
}

\section{The demands of modern photography have led to the evolution of extremely sensitive photographic emulsions. The vital role of gelatin in the emulsion is easily overlooked.}

IN the emulsion of a photographic film, silver chloride and bromide darken and decompose into their constituent parts when exposed to actinic light. This darkening is a very slow process unless it is accelerated chemically by use of a reducing solution or 'developer', which liberates silver from the exposed grains without affecting unexposed grains. This process furnishes most of the energy necessary to obtain an image. It is worth considering how an emulsion is produced, because the properties of the emulsion, almost always containing gelatin as the major colloid, are crucial in determining the quality of the image obtained.

\section{Emulsion manufacture}

The initial step in the manufacturing process involves the precipitation of silver chloride or bromide crystals from solutions of alkaline bromides and silver nitrate. The reaction takes place in a colloidal medium in order to keep the insoluble crystals of the silver salts in suspension.

The emulsion then undergoes a period of physical ripening or ageing during which the crystals grow larger. Any soluble salts remaining in solution are then removed by washing. For many years this involved gelling of the colloidal suspension and dialysis of the soluble salts by washing the gel in cold water. Today, most manufacturers rely on flocculation of the colloid by salts or organic reagents, including the modified gelatins, which flocculate in acid conditions.

Next comes a second, or chemical ripening. At this stage certain critical additions are made to encourage the formation of sensitivity centres on the crystals of the silver salts.

Finally, various additives are used to stop the ripening process, to encourage the coating of the emulsion and to harden the emulsion and make it insoluble to the developer. The emulsion is then coated into its support and cooled. It is coated with a protective layer of gelatin which is photographically inert.

\section{Role of gelatin}

Gelatin is generally the colloid of choice for the manufacture of emulsions. During precipitation and physical ripening it keeps the silver salts in suspension and is strongly absorbed at the surface of the silver halide crystals, retarding their growth. The ability of gelatin to form a gel allows the elimination of unwanted soluble salts by dialysis, and its high viscosity facilitates the coating of the emulsion onto the support.
After coating, the gel formed keeps the silver salt crystals on the support during drying and development, and when the emulsion is exposed to light, the gelatin prevents the inverse reaction of the photolysis by fixing the liberated halogen. Gelatin also prevents fading of the latent image resulting from the photolysis.

During development, the swelling of the gelatin, caused by the action of the added reagents, allows the reagents to diffuse through the emulsion layer. The property of the gelatin to be tanned by various substances allows rapid development at high temperatures.

Since gelatin is extracted from the collagen of bone or skin, most preparations inevitably contain impurities. Some are detrimental to the photographic process and must be removed (dust, heavy metals, grease and 'fogging agents'). Albumens, polypeptides containing cystine and nucleic acids act as 'restrainers', blocking the formation of sensitivity sites or inhibiting the development of silver halide grains. Some impurities, however, have useful properties acting as 'sensitizers' and facilitating the production of silver or silver sulphide centres on the halide grains.

Photographic gelatins can be classified into four groups: Inert gelatin, without sensitizers or restrainers; active gelatin, rich in sensitizer and low in restrainer; retarded gelatin, low in sensitizer and rich in restrainer; and retarded active gelatin, rich in both sensitizer and restrainer.

The task of the photograhic emulsion manufacturer is to find the best gelatin for a specific application. The gelatin manufacturer must produce the gelatin to an exact specification and ensure that quality is maintained from batch to batch.

\section{Gelatin production}

The conditions under which gelatin extraction occurs determine the properties of the end product. In particular, the liming process has to be closely controlled. Chemical changes occurring at this stage include the hydrolysis of amide groups to carboxylic groups (destroying the triple helix of collagen), the elimination of many impurities (mucopolysaccharides, tyrosine, sulphur), the transformation of the sulphur in cystine to thiosulphate and the degradation of ribonucleic acid to nucleotides.

After liming, the raw material is washed with water to eliminate as much lime as possible, and then 'delimed' in a bath of dilute acid. Gelatin is then extracted in hot water, with only the first extraction pure enough for the exacting requirements of photography.

\section{Modified gelatins}

It is possible to chemically modify gelatins in order to provoke floculation simply by lowering the $p H$. This type of gelatin is increasingly used for floculation of emulsions, replacing sodium or ammonium sulphate or washing by dialysis.

\section{Auxiliary gelatins}

Photographic materials do not consist of a single light sensitive layer. Other layers -auxiliaries-are coated onto the support before, or at the same time as, the emulsion layer. The support itself is usually synthetic and its surface has little affinity for the hydrophilic emulsion. An intermediate layer or substratum is required, containing the necessary ingredients to establish a bond between the emulsion and support.

Colour films or papers contain several emulsion layers, each sensitive to a different radiation band. The layers must be separated by intermediate layers to prevent diffusion from one colour layer to another.

The surface of the emulsion layer must be protected mechanically from external contact. This is the role of the supercoat. Like all auxiliary layers, it must have no photographic influence on the emulsion. They are commonly inert gelatines.

In order to compensate for the tension created on one side of the support by all the layers, a backing layer is coated on the other side of the support. This is often coloured in order to avoid the halo created by the light ray as it reflects on the film. Some colour films can contain as many as a dozen layers of chemicals. The sophisticated coating machines in use today allow the simultaneous coating of most of the layers at speeds of 100 to 150 metres per minute. Each layer is only some $10 \mu \mathrm{m}$ thick, so it is easy to appreciate the importance of the physical qualities required of the gelatin.

To supply the customer with a photographic gelatin with precisely the properties required, the manufacturer must be able to control the production process very accurately, and quality control must be stringent. In addition, it is useful for the customer to appreciate the problems of the manufacturer when specifying requirements for an emulsion.

Yves Lestra is an engineer at the head office of Rousselot S.A., 8 Rue Christophe Colomb, 75008 Paris, France. 